\title{
Correcting coils in end magnets of accelerators
}

\author{
L. R. P. Kassab \\ Faculty of Technology, Centro Estadual de Educação Technológica “Paula Souza”-UNESP, Praca Coronel Fernando Prestes 30, \\ CEP 01124-060, São Paulo, SP, Brazil \\ P. Gouffon \\ Institute of Physics, University of São Paulo, São Paulo, Brazil
}

(Received 9 March 1998; published 14 May 1998)

\begin{abstract}
We present an empirical investigation of the correcting coils behavior used to homogenize the field distribution of the race-track microtron accelerator end magnets. These end magnets belong to the second stage of the $30.0 \mathrm{MeV} \mathrm{cw}$ electron accelerator under construction at IFUSP, the race-track microtron booster, in which the beam energy is raised from 1.97 to $5.1 \mathrm{MeV}$. The correcting coils are attached to the pole faces and are based on the inhomogeneities of the magnetic field measured. The performance of these coils, when operating the end magnets with currents that differ by $\pm 10 \%$ from the one used in the mappings that originated the coils copper leads, is presented. For one of the magnets, adjusting conveniently the current of the correcting coils makes it possible to homogenize field distributions of different intensities, once their shapes are practically identical to those that originated the coils. For the other one, the shapes are changed and the coils are less efficient. This is related to intrinsic factors that determine the inhomogeneities. However, we obtained uniformity of $0.001 \%$ in both cases. [S1098-4402(98)00002-0]
\end{abstract}

PACS numbers: $41.85 . \mathrm{Lc}$

\section{INTRODUCTION}

The quality of the electron beam depends on the magnetic field distribution of the end magnets that deflect, focus, and return the beam to the accelerating section. Their uniformity must be better than $\Delta B / B= \pm 10^{-3}$ in order to keep the synchronism [1] between the electron beam and the accelerating microwave. The method of correction employed to homogenize the IFUSP race-track microtron booster accelerator magnets assured uniformity $[2,3]$ of $10^{-5}$ in an average field of $0.1 \mathrm{~T}$, over an area of $700 \mathrm{~cm}^{2}$. This method uses the principle of attaching to the pole faces correcting coils with copper leads, whose shapes are based on the inhomogeneities of the magnetic field measured [4]. When the inhomogeneous magnetic field has tangential components that are symmetrical to the middle plane, there are calculated planes that enable the construction of the correcting coils that are more efficient than those based on the mappings performed at the pole faces. The best result [5] is obtained for planes situated at $\pm 60 \%$ of the half gap height from the middle plane. Several tests were done to investigate the behavior of the inhomogeneities and the performance of the correcting coils [6] when operating the magnets with currents that differ by $\pm 10 \%$ from the one $(27.4 \mathrm{~A})$ that was used in the mappings that originated the correcting coils.

\section{EXPERIMENTAL PROCEDURE}

The inhomogeneities in both magnets are practically symmetrical to the middle planes. So, for each magnet, we did two identical double sided correcting coils, made of etched printed circuit boards, based on the arithmetic mean of four field maps [3], two $12 \mathrm{~mm}$ above and two $12 \mathrm{~mm}$ below the middle plane $( \pm 60 \%$ of $20 \mathrm{~mm}$, the half gap height), and placed them at the pole faces. The copper leads $\left(4 \times 10^{-5} \mathrm{~m}\right.$ thickness $)$ were shaped like the lines of equal magnetic field obtained after this procedure. The mapping of each plane was performed with a pair of differential Hall probes $( \pm 1.5 \mu \mathrm{T}$ resolution), in 1170 points separated by a distance of $8 \mathrm{~mm}$, using $27.4 \mathrm{~A}$ for the current of operation. One of the probes was maintained fixed in the middle plane, near the magnet edge, and the other one was attached to an automated mechanical system that moved it in a plane of the magnet gap.

The magnets were submitted to a well-defined cycling procedure, empirically determined, that provided reproducibility of $10^{-5}$ for a magnetic field distribution of about $0.1 \mathrm{~T}$. Stability of the same order was also observed during 9 hours. Figures 1 and 2 show the two magnets correcting coils.

\section{THE PERFORMANCE OF THE CORRECTING COILS}

Figures 3 and 4 show field distributions in the middle plane between the pole faces of the magnets operated at $27.4 \mathrm{~A}$ (the points indicate the coordinates, in millimeters, of each magnetic field difference measurement). The magnets exhibit differences in terms of the shape and magnitude of their homonogeneities. In the first one $\Delta B / B=$ $\pm 1.4 \times 10^{-3}$, and in the other $\Delta B / B= \pm 6 \times 10^{-4}$.

The optimal currents for the correcting coils of each magnet, $122.2 \mathrm{~mA}$ for one of them and $115.7 \mathrm{~mA}$ for 


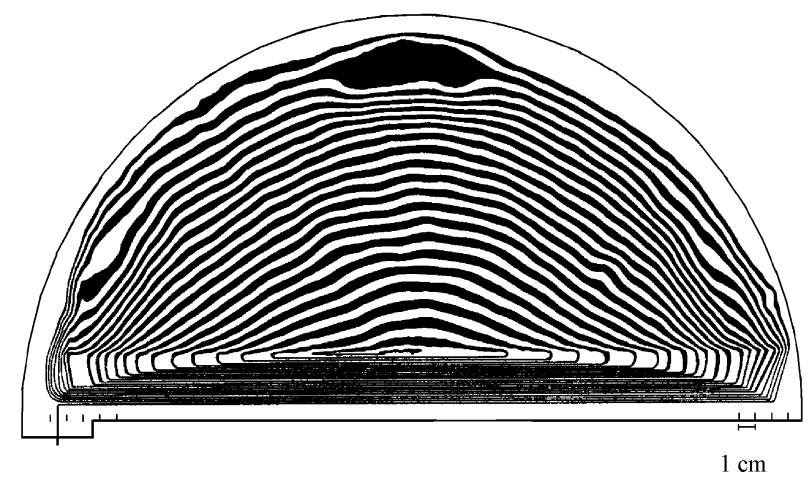

FIG. 1. The correcting coils used for the first magnet. The interval between the copper leads is $7.4 \mu \mathrm{T}$.

the other one, were found empirically and are in good agreement with the theoretical value $(117.8 \mathrm{~mA})[2,6]$.

The standard deviation, $65.1 \mu \mathrm{T}$, for the field distribution in the middle plane of the first magnet, shown in Fig. 3, becomes $1.8 \mu \mathrm{T}$ (Fig. 5), using the optimal current value $122.2 \mathrm{~mA}$ for the correcting coils and $27.4 \mathrm{~A}$ for the current of operation. Figure 4 shows the field distribution in the middle plane of the second magnet operated at 27.4 A, in which the standard deviation, $17.8 \mu \mathrm{T}$, becomes $2.5 \mu \mathrm{T}$ (Fig. 6, using the optimal value $115.7 \mathrm{~mA}$ for the correcting coils.

For the first magnet, although the shape of the inhomogeneity changes almost nothing when the current of operation is altered, there is variation in its intensity. Then we adjusted conveniently the current of the correcting coils, in order to compensate this effect. When the current of operation is raised from 27.4 to $30.0 \mathrm{~A}$, the correction done by $122.2 \mathrm{~mA}$ current becomes increasingly excessive, as can be seen by the growth of the standard deviations (Fig. 7). Then, for the correcting coils, we used currents successively smaller and always lower than $122.2 \mathrm{~mA}$ (Fig. 8). Comparatively, when the current of operation is raised from 24.7 to $27.4 \mathrm{~A}$, the correction performed by the $122.2 \mathrm{~mA}$ current becomes decreasingly insufficient, as shown by the lessening of the standard deviations (Fig. 7). This explains the need of currents successively smaller but always higher than $122.2 \mathrm{~mA}$ (Fig. 8) to compensate this effect. The results obtained for this magnet lead us to conclude that the field distributions, before the corrections, for currents of operation higher than $27.4 \mathrm{~A}$, are closer to those that originated the correcting coils and of smaller variation. Figure 7 reveals the performance of the coils in the exposed situations, in which were obtained uniformity of $10^{-5}$, as the standard deviation does not exceed $3.1 \mu \mathrm{T}$.

On the other hand, for the second magnet, when the current of operation is altered, the shape of the inhomogeneity changes, especially for current smaller than 27.4 A (this can be seen comparing Figs. 9 and 10 with Fig. 4). Even so, we adjusted the current of the correcting coils to optimize the results. Nevertheless, we found few differences between the results (Fig. 11), mainly for currents smaller

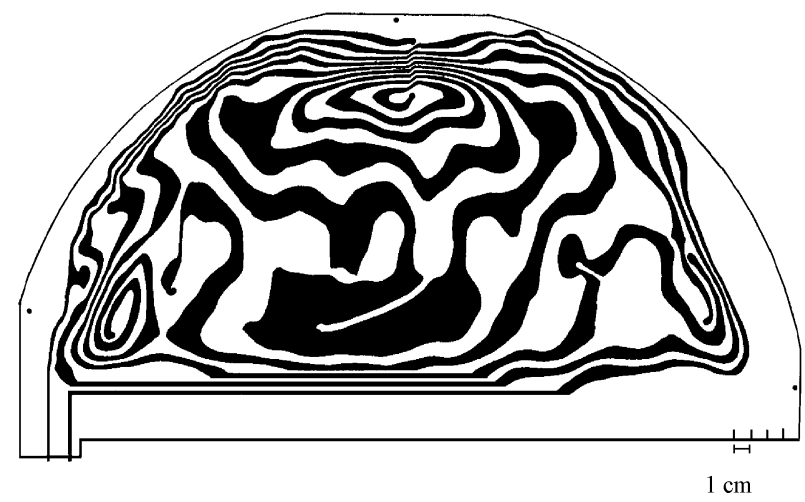

FIG. 2. The correcting coils used for the second magnet. The interval between the copper leads is $7.4 \mu \mathrm{T}$.

than $27.4 \mathrm{~A}$, where the standard deviations are practically superimposed. When the magnet is operated with currents greater than $27.4 \mathrm{~A}$, the distributions of the field difference measurements are more similar to those that originated the correcting coils, but of greater variation. This explains the achievement of the best corrections revealed by the standard deviations (Fig. 11) and the need to increase the correcting coils current in the interval of operation (Fig. 12). Contrarily to the other magnet, in this case, the shape of the correcting coils copper leads does not exactly correspond to the inhomogeneities that have to be compensated. This is why the correction performed by the coils is less efficient. However, the uniformity obtained is of about $10^{-5}$.

\section{CONCLUSIONS}

The magnets present inhomogeneities that differ in terms of their shapes and strengths that lead them to behave differently when the current of operation is altered.

Through the results obtained, we come to the conclusion that the success of the method of correction employed is related to the fact that the correcting coils must represent copies of the inhomogeneities. The results obtained for the first magnet show that, when the current of operation is varied by $\pm 10 \%$, the shape of the inhomogeneities is almost not altered and the intensity change can be compensated adjusting properly the correcting coils current. For the second magnet, the change in the inhomogeneity shape compromises the performance of the correcting coils. Besides, we should add that the first magnet, with a well-defined inhomogeneity, in spite of being initially less uniform, is more susceptible to the correction but less susceptible to changes in the shape of the field distribution. This suggests that a greater uniformity represents a greater tendency for changes in the field distribution when the current of operation is altered. These effects are related to intrinsic factors that determine the inhomogeneities. However, both of the recirculating magnets present uniformity of $10^{-5}$ when operated in the interval studied. 


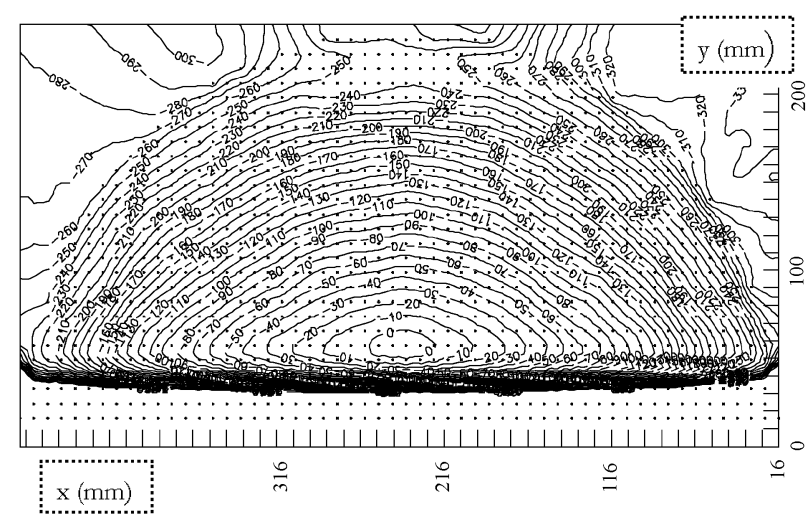

FIG. 3. Field distribution in the middle plane of the first magnet before the correction, using 27.4 A for the current of operation. Difference between two lines is $10 \mu \mathrm{T}$.

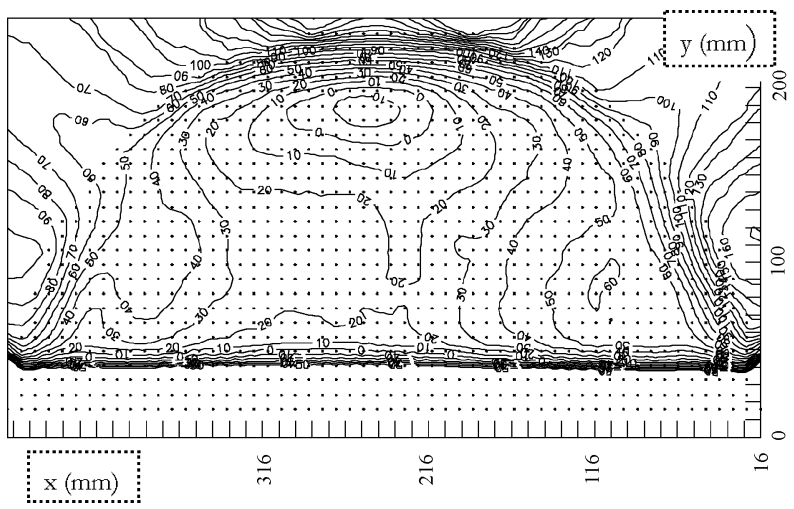

FIG. 4. Field distribution in the middle plane of the second magnet before the correction, using $27.4 \mathrm{~A}$ for the current of operation. Difference between two lines is $10 \mu \mathrm{T}$.

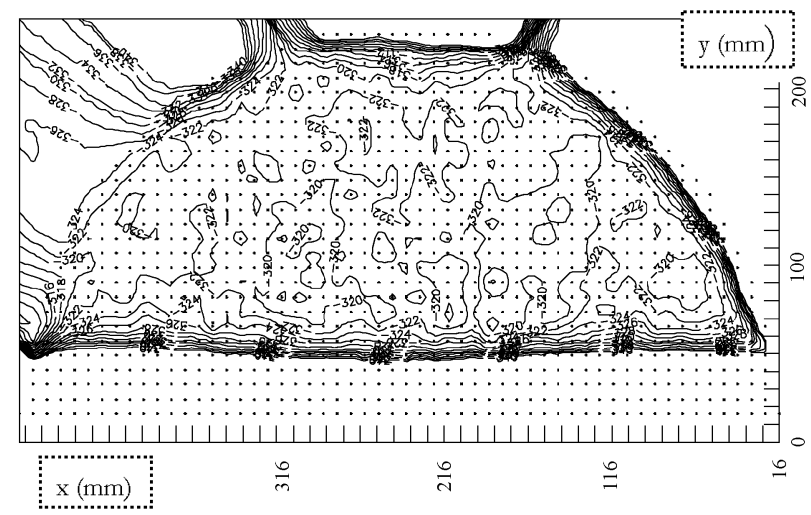

FIG. 5. Field distribution in the middle plane of the first magnet using for the current of operation 27.4 A and for the correcting coils the optimal current (122.2 mA). Difference between two lines is $2 \mu \mathrm{T}$.

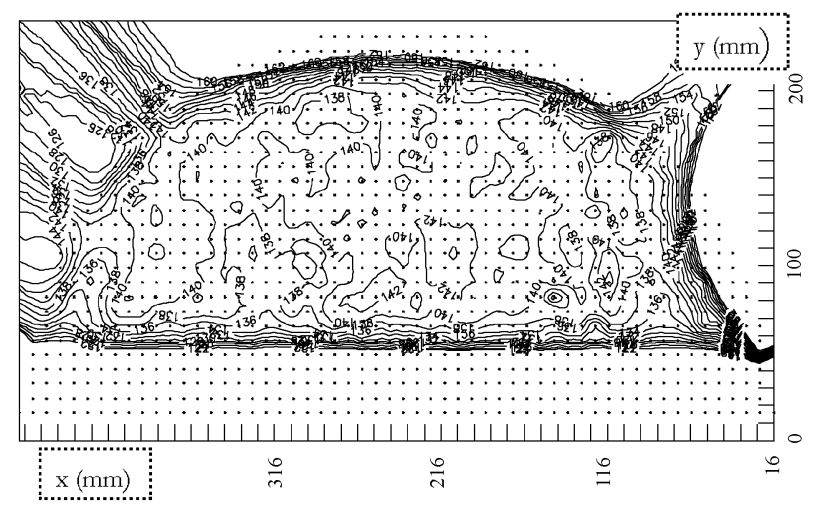

FIG. 6. Field distribution in the middle plane of the second magnet using for the current of operation 27.4 A and for the correcting coils the optimal current (115.7 mA). Difference between two lines is $2 \mu \mathrm{T}$.

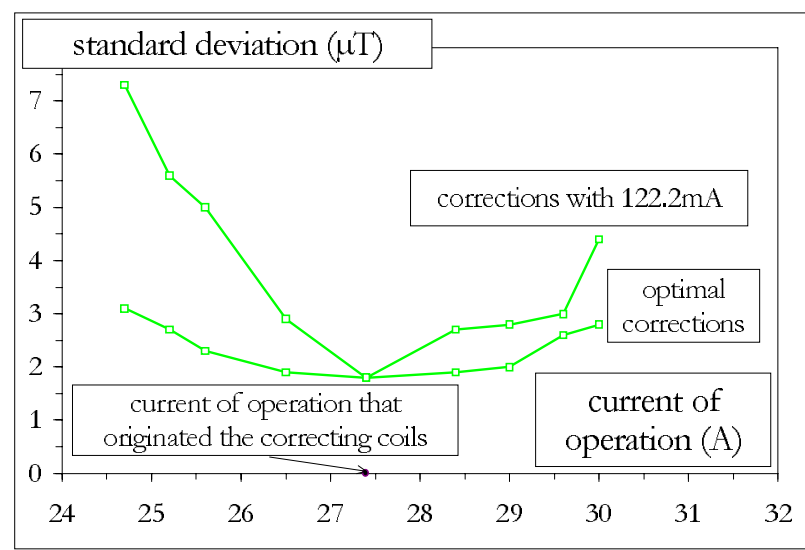

FIG. 7. (Color) Standard deviations of field difference measurements for different currents of operation (first magnet).

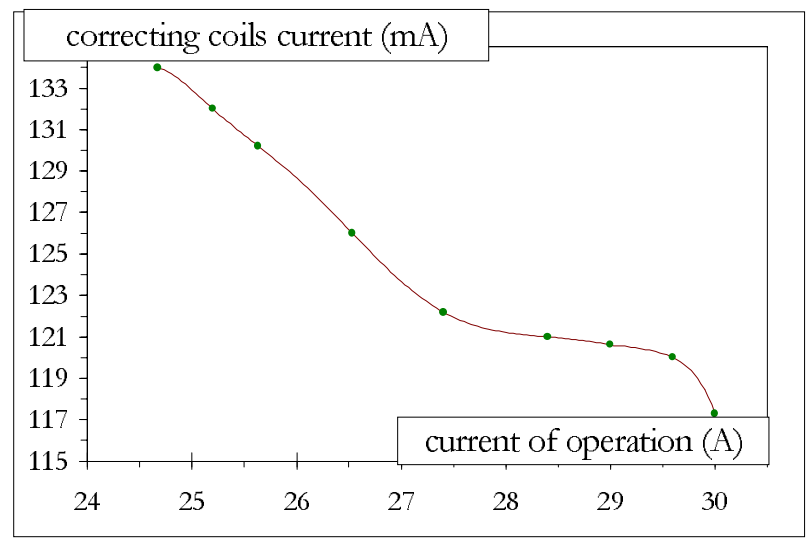

FIG. 8. (Color) Correcting coils currents for different currents of operation (first magnet). 


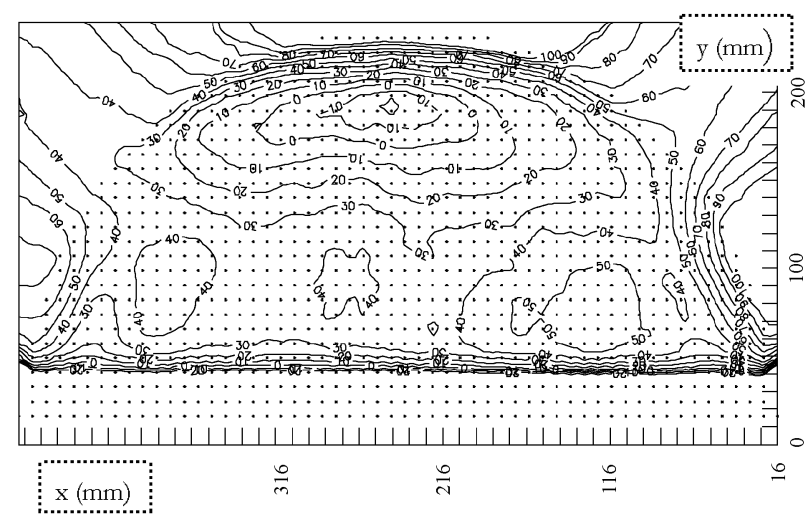

FIG. 9. Field distribution in the middle plane of the second magnet before the correction, using 24.7 A for the current of operation. Difference between two lines is $10 \mu \mathrm{T}$.

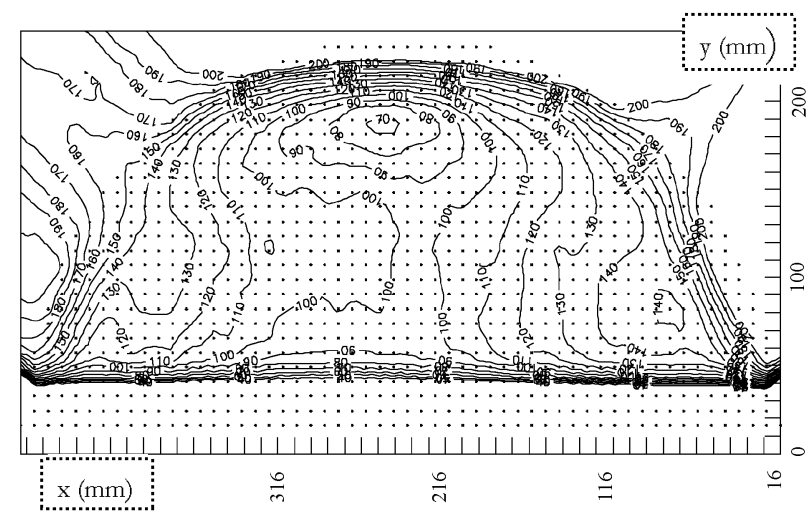

FIG. 10. Field distribution in the middle plane of the second magnet before the correction, using $30.0 \mathrm{~A}$ for the current of operation. Difference between two lines is $10 \mu \mathrm{T}$.

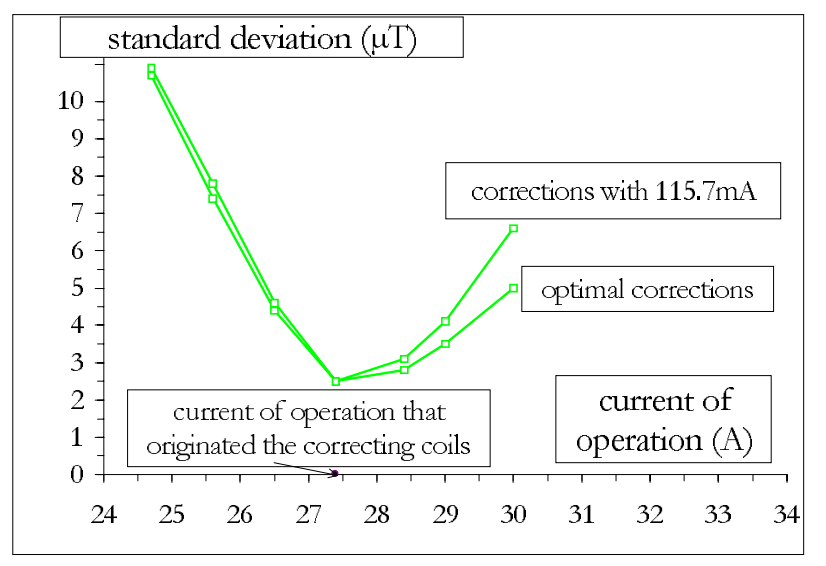

FIG. 11. (Color) Standard deviations of field difference measurements for different currents of operation (second magnet).

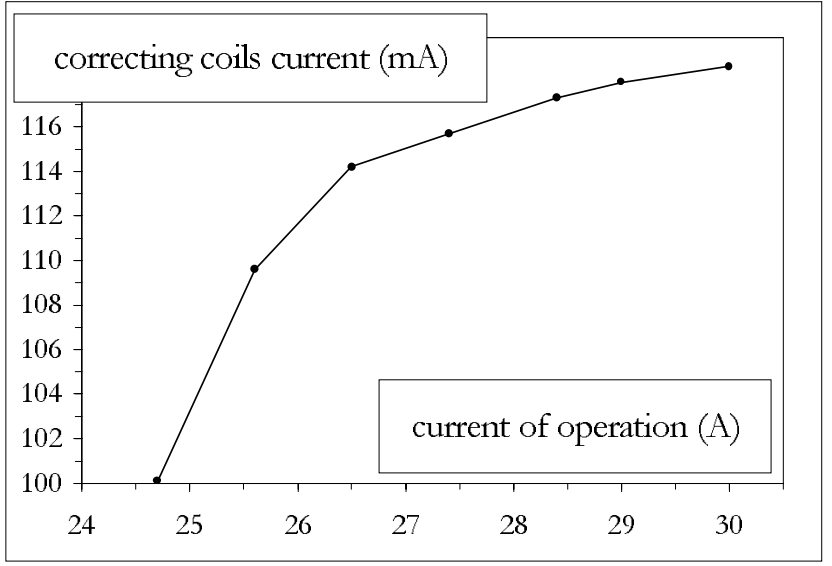

FIG. 12. Correcting coils currents for different currents of operation (second magnet).

\section{ACKNOWLEDGMENTS}

We express our thanks to Dr. Marcello Damy de Souza Santos, Dr. Giorgio Moscati, Dr. Maria V.P. Heller, and Dr. Ursula Ludwig Mertin for important suggestions during this work, as well as to Dr. Manoel T.F. Cruz, A. Malafronte, J. C. A. Alcon, and E. Monteiro for having gently developed the automated system used to map the end magnets. We also express our thanks to Dr. Marcos N. Martins, manager of the race-track microtron project, and Dr. Paulo Pascholati, coordinator of the IFUSP Linear Accelerator Laboratory, in the accomplishment of this work. We thank Faculty of Technology (FATEC-SP) for its encouragement during this work. We acknowledge financial support from FAPESP, CAPES, CNPQ, and FINEP.

[1] L. R. P. Kassab, P. Gouffon, M. N. Martins, and J. Takahashi, Particle Accelerators (to be published).

[2] L. R. P. Kassab, P. Gouffon, and M. N. Martins, Nucl. Instrum. Methods 404, 181 (1998).

[3] L. R.P. Kassab, Ph.D. thesis, University of São Paulo, 1996.

[4] U. Czok, G. Moritz, and H. Wollnik, Nucl. Instrum. Methods 140, 135 (1977).

[5] J. Friedrich and L. Tiator, Universitat Mainz, 1977.

[6] L. R. P. Kassab and P. Gouffon, Proceedings of the 1997 Particle Accelerators Conference, Vancouver, Canada (to be published). 